\title{
Vortices induced in a superconducting loop by asymmetric kinetic inductance and their detection in transport measurements
}

\author{
G. R. Berdiyorov, M. V. Milošević, and F. M. Peeters* \\ Departement Fysica, Universiteit Antwerpen, Groenenborgerlaan 171, B-2020 Antwerpen, Belgium
}

(Received 19 January 2010; published 21 April 2010)

\begin{abstract}
Using time-dependent Ginzburg-Landau theory, we study the dynamic properties of a rectangular superconducting loop, which are found to depend on the position of the current leads. For asymmetric positioning of the leads, different kinetic inductance of the two paths for injected electric current leads to different critical conditions in the two branches. System self-regulates by allowing vortex entry, as vortex currents bring equilibration between the two current flows and the conventional "resistive" state can be realized. We also demonstrate that individual vortex entry in the loop can be detected by measuring the voltage between normalmetal leads, for applied currents comparable in magnitude to the screening currents.
\end{abstract}

DOI: 10.1103/PhysRevB.81.144511

PACS number(s): 74.78.Na, 73.23.-b

\section{INTRODUCTION}

The quantization of magnetic flux ${ }^{1}$ is one of the most fundamental properties of conventional superconductors, which nicely explains experiments on, e.g., weakly connected rings, ${ }^{2}$ Little-Parks oscillations, ${ }^{3}$ and enhancement of critical parameters of multiply connected superconductors. ${ }^{4}$ The total magnetic flux $\Phi$ through a superconducting area, enclosed by a contour of zero current is an integer multiple of the flux quantum $\Phi_{0}=h c / 2 e$. In contrast to bulk superconductors, in mesoscopic samples current is not zero at the sample boundary but it changes polarity at an effective radius $\rho^{*}$ inside of which the flux is exactly quantized. ${ }^{5}$ The zero-current path however follows the shape of the sample boundary and is responsible for the peculiarities of the vortex matter in mesoscopic samples. ${ }^{6}$

Besides the flux quantization, the profile of superconducting currents in the sample is important for fluxoid dynamics (e.g., the maxima of current in mesoscopic samples predetermine the weak points for vortex entry) which was the subject of a number of experimental ${ }^{7-9}$ and theoretical works. ${ }^{10,11}$ Applied electrical current to the sample further modifies the overall current profile, subjects vortices to a Lorentzian drive, and can lead to phase-slip phenomena and the "resistive" state. ${ }^{12}$ This is well understood for symmetric samples, particularly rings and stripes, but the complexity of the problem rises if asymmetry is introduced.

Over 30 years ago, de Waele et al. ${ }^{13}$ constructed an asymmetric superconducting loop, consisting of a niobium foil and a tin needle. They measured the nonzero voltage as a function of the enclosed magnetic flux even at zero external current. Recently, similar results were reported in Ref. 14, where the voltage was measured across a symmetric aluminum loop. These results were explained by thermal fluctuations and electrical noise near the critical temperature. ${ }^{15} \mathrm{By}$ introducing an asymmetry by varying the width $\left(w_{1,(2)}\right)$ of one of the Al loop arms, authors of Ref. 16 reported a shift $\Delta \Phi$ in Little-Parks oscillations for different polarity of the injected current, with a maximal shift of $0.5 \Phi_{0}$ for asymmetry ratio $w_{1} / w_{2}$ above 1.25 .

In this paper, we investigate the flux quantization, vortex entry, and the repercussions of the interplay of Meissner and applied currents in a specially designed rectangular superconducting loop. The sample is biased symmetrically and asymmetrically through the attached normal leads, i.e., asymmetry is introduced by varying the length of the loop arms (see Fig. 1). This provides a different kinetic inductance of the two loop arms, resulting in proportional splitting of applied current into two branches, and leads to asymmetric critical phenomena in the loop. The consequent selfregulation of the system is the main topic of our further analysis.

\section{APPROACH USED}

We consider a thin rectangular superconducting loop (with thickness $d$ much smaller than the coherence length $\xi$ and the penetration depth $\lambda$, and with lateral sizes $a$ and $b$ and width $w$ ) with a transport electric current applied through the normal contacts (size equal to the loop width) in the presence of a magnetic field $H$ perpendicular to the plane of the loop (see Fig. 1). For the given system, we numerically solved the time-dependent Ginzburg-Landau (GL) equation which can be written in the following form close to the superconducting-normal $(\mathrm{S} / \mathrm{N})$ transition temperature $T_{c} \cdot{ }^{17}$

$$
\begin{aligned}
& \frac{u}{\sqrt{1+\Gamma^{2}|\psi|^{2}}}\left(\frac{\partial}{\partial t}+i \varphi+\frac{\Gamma^{2}}{2} \frac{\partial|\psi|^{2}}{\partial t}\right) \psi=(\nabla-i \mathbf{A})^{2} \psi \\
& +\left(1-|\psi|^{2}\right) \psi .
\end{aligned}
$$

This equation is coupled with the equation for the electrostatic potential, (a)

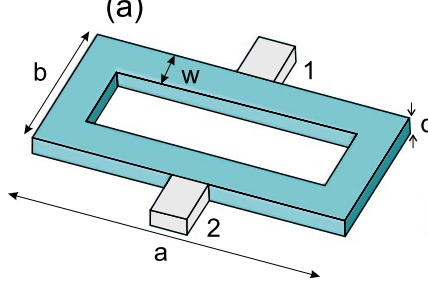

(b)

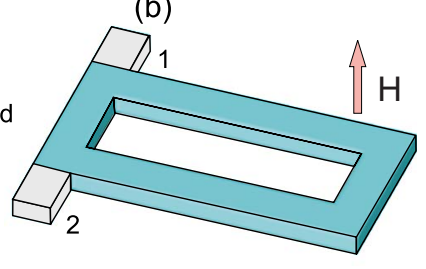

FIG. 1. (Color online) Schematic view of the studied system: a rectangular superconducting loop (dimensions $a \times b$, arm width $w$, thickness $d$ ) with dc current injected through depicted leads in the (a) symmetric and (b) asymmetric case. 


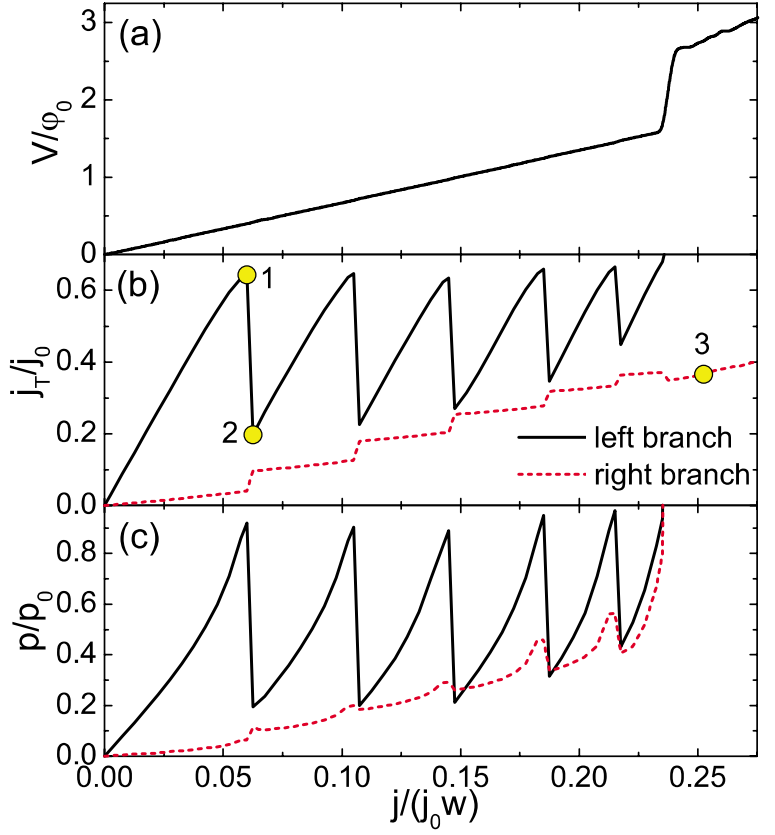

FIG. 2. (Color online) (a) The time-averaged voltage, (b) the total current density, and (c) the supervelocity as a function of applied current density for the sample of dimensions $a=32 \xi, b=16 \xi$, and $w=2 \xi$ with a defect of size $1.5 \times 1 \xi$ Indicated states (1-3) are shown in Fig. 3.

$$
\Delta \varphi=\operatorname{div}\left\{\Im\left[\Psi^{*}(\nabla-i \mathbf{A}) \Psi\right]\right\},
$$

which is nothing else than the condition for the conservation of the total current in the system, i.e., div $\mathbf{j}=0$. Here, distance is scaled to the coherence length $\xi$, time is in units of the GL relaxation time $\tau_{\mathrm{GL}}=\pi \hbar / 8 k_{B} T u$, the electrostatic potential $\varphi$ is scaled to $\varphi_{0}=\hbar / 2 e \tau_{\mathrm{GL}}$, and vector potential $\mathbf{A}$ is scaled by $H_{c 2} \xi$. The parameter $\Gamma=2 \tau_{E} \Psi_{0} / \hbar$ characterizes the chosen superconducting material (with $\tau_{E}$ being the inelastic electron-collision time and $\Psi_{0}$ is the value of the order parameter at zero applied field and current). The material parameters $u$ and $\Gamma$ are taken as $u=5.79$ and $\Gamma=20$, realistic for low- $T_{c}$ samples. ${ }^{17}$ Neumann boundary condition is used at all sample boundaries, except at the current contacts ( $\mathrm{S} / \mathrm{N}$ junction) where we used $\psi=0$ and $\left.\nabla \varphi\right|_{n}=-j$ with $j$ being the applied current density in units of $j_{0}=c \Phi_{0} / 8 \pi^{2} \lambda^{2} \xi$. Note that in Eq. (1), the screening of the magnetic field is neglected due to the very small sample thickness $(d \ll \xi, \lambda)$. We solved the thickness averaged Eqs. (1) and (2) self-consistently, using standard finite-difference iterative methods.

\section{CURRENT-INDUCED VORTICES IN ASYMMETRIC LOOPS}

Figure 2(a) shows the $I-V$ curve of the asymmetric sample with dimensions $a=32 \xi, b=16 \xi$, and $w=2 \xi$ without applied magnetic field. With increasing the applied current $j_{21}$ (which flows from contact 2 to contact 1, see Fig. 1), the system is in full superconducting state [Fig. 3-1] until the critical current $j_{c 2}$ is reached, where the system transits to a resistive state with a finite-voltage jump. This resistive state is character-

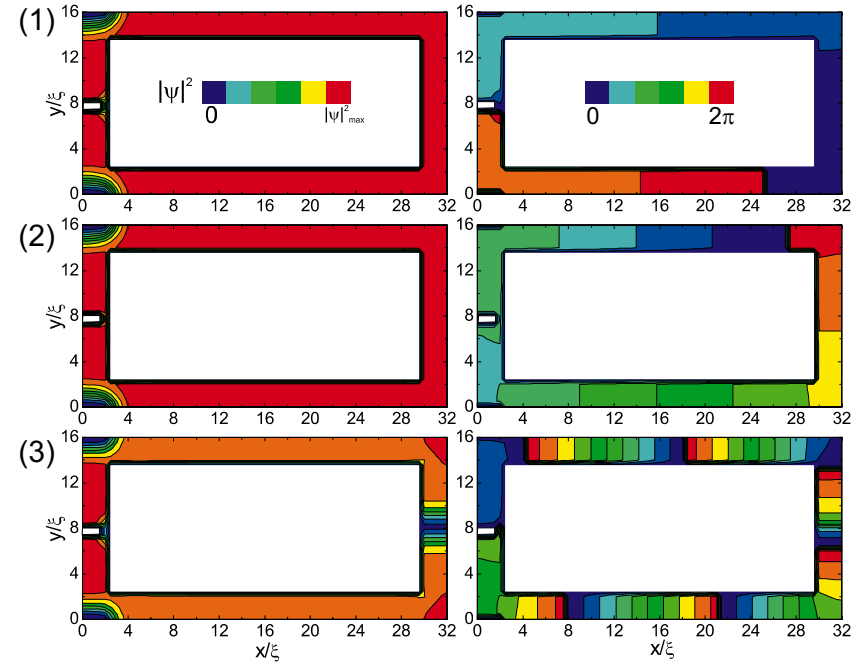

FIG. 3. (Color online) Contour plots of the Cooper-pair density (left column) and the phase of the order parameter (right column) at the values of the applied current indicated by open (yellow) dots in Fig. 2(b).

ized by the periodic entrance of fast-moving vortexantivortex pairs, in a channel with strongly suppressed order parameter [see Fig. 3-3]. ${ }^{18}$

The total current density $j_{T}=j_{S}+j_{n}$ [where $\mathbf{j}_{S}=\left(\psi^{*} \nabla \psi\right.$ $\left.-\psi \nabla \psi^{*}\right) / 2 i-|\psi|^{2} \mathbf{A}$ is the supercurrent density and $\mathbf{j}_{n}=-\nabla \varphi$ is the normal current density] measured in the middle of the short arms of the loop and averaged over the loop width $w$ is shown in Fig. 2(b) as a function of the applied current density $j$. Due to the asymmetric length of the current paths in our sample, the current density in the left branch $j_{l}$ reaches the critical value $j_{c 2}$ first. At this point, vortices are bound to nucleate in the loop, in order to decrease the current in the short (left) arm [see Fig. 3-2]. In turn however, this should result in an increase in the current in the right arm, due to simple superposition, and equilibration of the system seems untrivial. Note that we introduced a defect (of size $1.5 \times 1 \xi$ ) in the left branch, as shown in Fig. 3, to facilitate the vortex entry in the loop. Typically, much larger flux jumps are found in large superconducting loops,${ }^{10}$ and we wanted to demonstrate more clearly the vortex nucleation due to asymmetry.

The current resulting from the incoming vortices is directed in such a way that it decreases abruptly the larger current density in the left arm and increases stepwise the one in the right branch [cf. Figs. 4(a) and 4(b)]. This means that the system self-regulates the current distribution by allowing vortex entry inside the loop. With further increasing applied current, $j_{l}$ increases faster than the current density in the right branch $j_{r}$ and reaches $j_{c 2}$ on multiple (five) occasions (accompanied by vortex entry) before the transition to the resistive state. However, even at the latter transition, $j_{l}$ is larger than $j_{r}$ [compare solid and dashed curves in Fig. 2(b)], and therefore the condition for the resistive state, occurring simultaneously in both arms, cannot be explained by the corresponding density of currents. To get the threshold condition, we use the momentum of the superconducting condensate (also called supervelocity) $\mathbf{p}=\nabla \theta-\mathbf{A}$, where $\theta$ is the phase of the order parameter. As shown in Fig. 2(c), $p$ is 


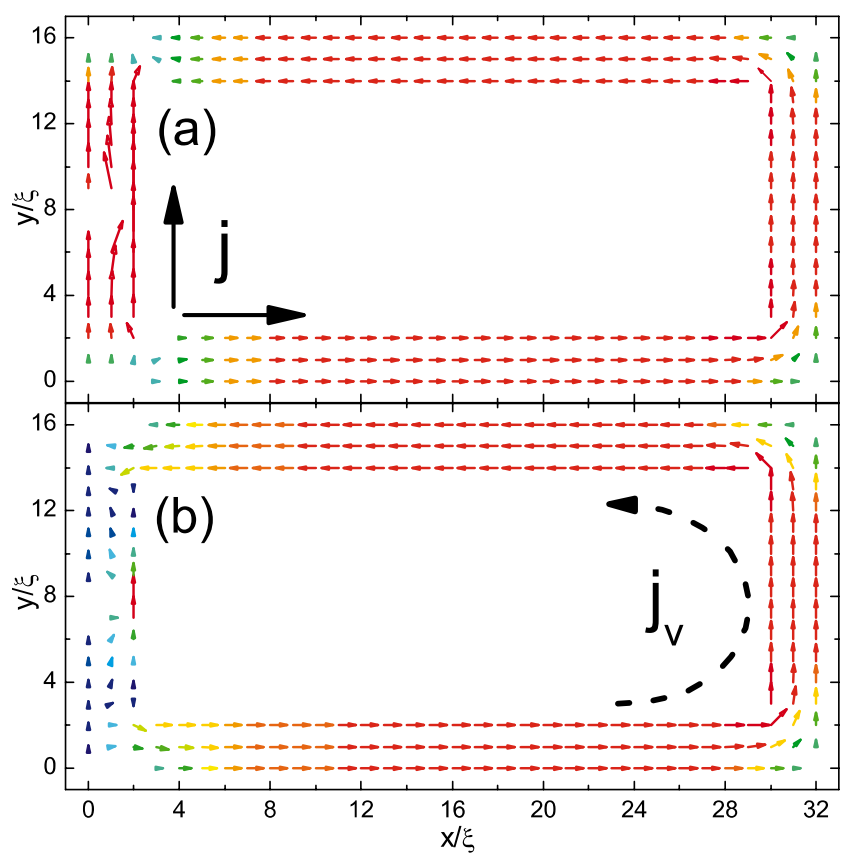

FIG. 4. (Color online) (a), (b) Vector plots of the supercurrent density for the values of applied current indicated by 1 and 2, respectively, in Fig. 2(b). The arrows depict the direction of the applied current $j$ and the vortex current $j_{v}$.

monotonously increasing with $j$ apart from the jumps when vortices enter the sample. Prior to the resistive state, the supervelocities in the two branches indeed become equal.

\section{EFFECT OF THE APPLIED MAGNETIC FIELD}

As we showed above, the superconducting ring can respond to external current by allowing penetration of quantized flux into the system. In what follows, we investigate the behavior of both symmetric and asymmetric samples in applied magnetic field. We do so by monitoring the voltage between the leads, which can reveal the subtle changes in the superconducting state.

\section{A. Determination of vortex states in transport measurements}

It is already known that the dependence of the local electric field on the magnetic field can be observed close to the transition to the normal state, as found in the original LittleParks experiment. ${ }^{3}$ The key for observable $V(H)$ features is the existence of $\mathrm{S} / \mathrm{N}$ domains and interfaces in the sample, which enables the survival of the normal current in the superconducting sample. Namely, the length over which the nonequilibrium (NQ) quasiparticles can exist in the sample $L_{n q}=\sqrt{D \tau_{E}}\left(D\right.$ being the diffusion constant $\left.{ }^{19}\right)$ is often larger than the size of the mesoscopic samples, and the voltage due to the normal current can be detected. In our case, key conditions for voltage observations are met thanks to the $\mathrm{S} / \mathrm{N}$ interfaces at the leads. However, one should note that the measured voltage due to NQ quasiparticles will depend on their entire path and Andreev recombinations at each $\mathrm{S} / \mathrm{N}$ interface in the sample, including vortices. As a consequence, not only can we monitor the evolution of vortex states with the magnetic field but we can also detect the geometry of the vortex state following the idea of Kanda et $a^{20}$ By comparing the voltages at strategically placed superconductor-insulator-normal (S/I/N) junctions, Kanda et al., could determine if the superconducting gap was uniform or not at the periphery of the sample, indicating the giantvortex and multivortex state, respectively. However, to distinguish between giant and multivortex states, ${ }^{11,21}$ we no longer need multiple $\mathrm{S} / \mathrm{I} / \mathrm{N}$ tunnel junctions on the sample. Instead we can simply compare the measured voltages at strategically placed normal contacts. Therefore, the proposed method is far simpler and useful for transport studies of both stationary and dynamic intermediate states in mesoscopic type-II as well as in type-I superconductors.

\section{B. Symmetrically placed contacts}

As a proof of the above discussion, Fig. 5(a) shows the calculated voltage $V$ between the symmetric contacts at a weak injected current $j=0.05 j_{0}$, as a function of applied magnetic field $H$ for the sample dimensions as in Fig. 2. Large flux through the sample $\left[3.1 \Phi_{0}\right.$, see top axis in Fig. $5(\mathrm{a})]$ is needed for the entry of the first vortex, leading to a jump in the measured voltage. Such large first penetration field is the consequence of the surface barrier, and a typical mesoscopic effect. With further increase in applied field, vortices consecutively enter the sample, all accompanied by finite-voltage jumps. For the given parameters of the sample, the period of the vortex entry with field $\Delta \Phi$ was slightly larger than $\Phi_{0}$. This suggests that the flux is exactly quantized not over the whole sample but over an effective area $S^{*}$ bounded by the zero-current path [for the illustration of the effective radius of the area $S^{*}$, see the inset of Fig. 5(b)].

The total current density $j_{T}$ in left (solid curve) and right (dashed curve) branch of the loop is shown in Fig. 5(b) as a function of the applied field. As expected, the current density in the left branch $j_{l}$ [where the Meissner currents flow in the same direction as the applied one, see panel (3) in Fig. 5] is larger than the one in the right branch $j_{r}$. In the Meissner state (before the first voltage jump), $j_{l}$ increases monotonously with $H$ while $j_{r}$ drops to zero at $H^{*}$ due to the compensation of the applied current by the Meissner currents in this arm. Beyond $H^{*}$, the $j_{r}(H)$ curve shows the same behavior as $j_{l}(H)$ [actually, $j_{r}(H)=j_{l}(H)-2 j_{M}\left(H^{*}\right)$ ]. Obviously, the value of $H^{*}$ depends on the injected current density $j$ and clearly increases with increasing $j$.

\section{Asymmetric case}

Next, we contrast the above results with the case of the asymmetrically placed contacts. Figure 6 shows $V(H)$ for the asymmetric sample for two directions of the applied current $j$. For the direction of the current $j_{12}$, the current in the right arm $j_{r}$ has the same direction as the Meissner currents and voltage increases almost linearly with $H$ (thin curve) whereas in the symmetric case, parabolic dependence of the voltage on the field was found [see Fig. 5(a)]. The first penetration field also becomes larger compared to the symmetric case. 


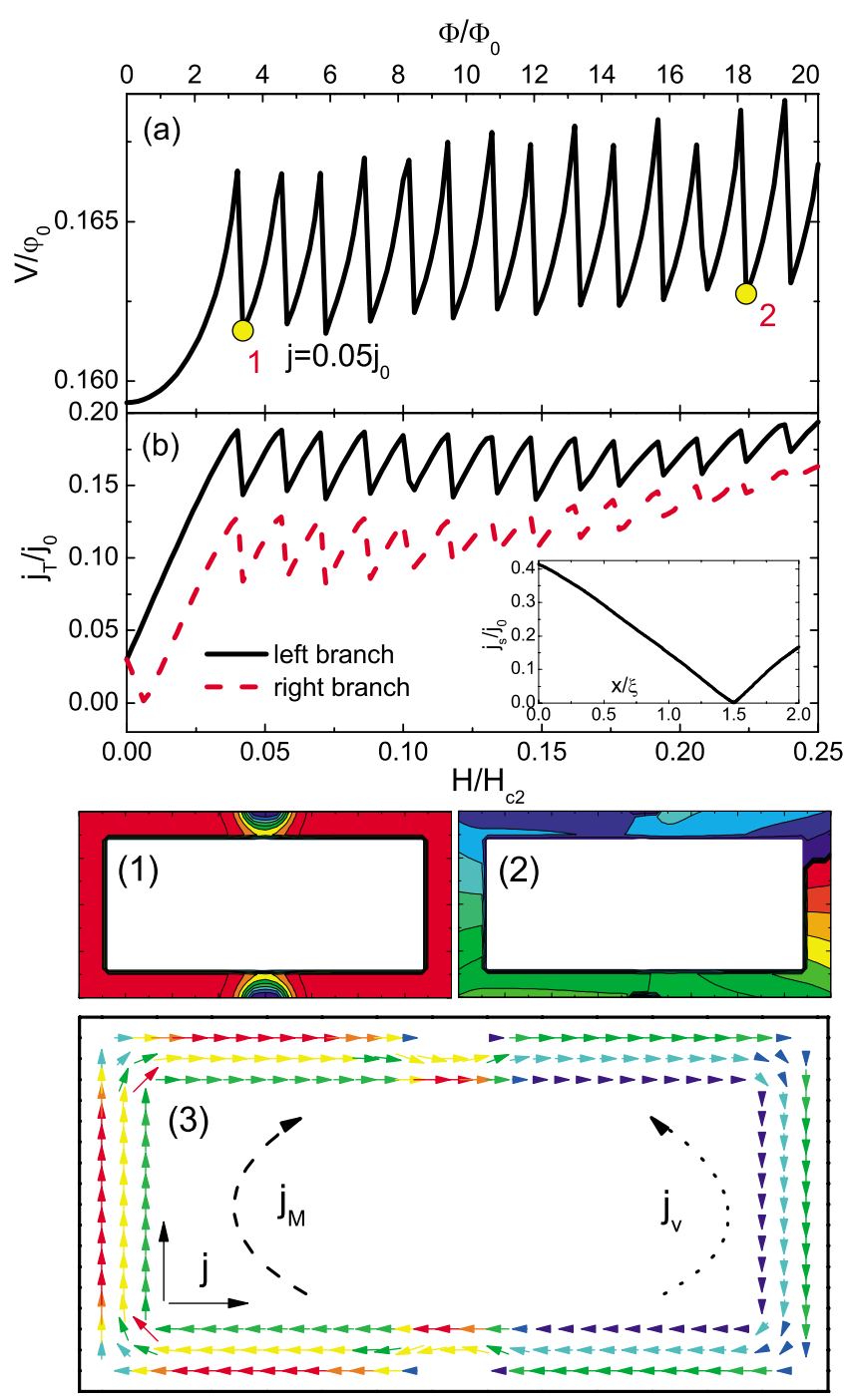

FIG. 5. (Color online) Vortex penetration in a symmetric sample with length $a=32 \xi$, width $b=16 \xi$, and the contact size $w=2 \xi$ : (a) calculated voltage between leads and (b) the total current density $j_{T}$ (averaged over the width $w$ ) along left (solid curve) and right (dashed curve) branch for injected current $j=0.05 j_{0}$, as a function of the applied magnetic field. The inset in (b) shows the amplitude of the supercurrent density $j_{s}$ across the loop, in the middle of the left arm at the field value indicated by 2 in (a). Panels (1)-(3) show the Cooper-pair density (1), phase of the order parameter (2), and supercurrent density (3) distributions for the $L=1$ state indicated in (a) by 1 . Arrows in (3) show the direction of the applied current $j$, and Meissner $j_{M}$ and vortex $j_{v}$ currents.

Upon each vortex entry, the vortex currents compensate the current density in the right arm and lead to a sharp decrease in the output voltage. At the same time, the voltage exhibits an overall decreasing tendency with increasing $H$.

When we alter the direction of the applied current (thick curve in Fig. 6), the following changes in $V(H)$ dependence are found: (i) voltage initially decreases with increasing the applied field and reaches a minimum at $\Phi \approx 3.1 \Phi_{0}$, where maximal compensation of the current in the right branch $j_{r}$ by the Meissner current is reached [see the directions of the currents in panel (3) of Fig. 5]. As a result, we observed a

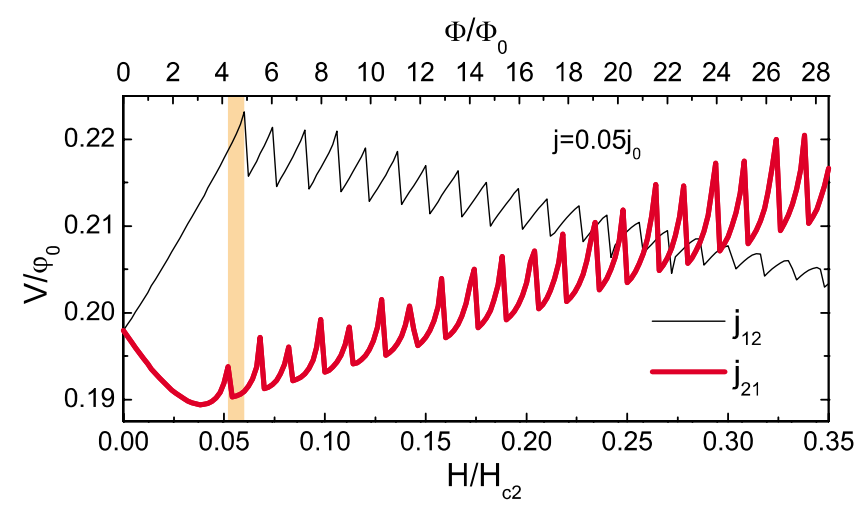

FIG. 6. (Color online) The same as in Fig. 5(a) but for the case of asymmetric biasing, and for two directions of applied current $\left(j=0.05 j_{0}\right): j_{12}$, thin (black) curves, and $j_{21}$, thick (red) curves. The shaded region highlights the difference in the transition fields between different vortex states for two opposite directions of the applied current.

shift in the transition field between different vortex states $\Delta \Phi_{L} \approx 0.6 \Phi_{0}$ for two polarities of asymmetric biasing (the offset is highlighted as a shaded region in Fig. 6). Note that for both directions of the applied current, the first penetration field was larger than the one in the symmetric case, although $j_{l}$ in the asymmetric case is always larger than the corresponding $j_{l}$ in the symmetric case. This proves that the penetration field is not solely determined by the local current density. (ii) The size of the voltage jumps increases with subsequent flux entry inside the loop. (iii) The voltage (and resistance) shows overall increasing tendency with increasing $H$ contrary to the case of $j_{12}$ biasing. The latter is related to the superconducting state of the right arm (where vortex currents are added to $j_{r}$ and gradually suppress superconductivity). In the case of asymmetric biasing, two arms act as two resistors tied in parallel-so that the electric response of the sample is mostly determined by the smaller resistance of the two, i.e., the larger loop arm. Similar behavior of the output voltage on the applied field has also been reported in experiments on asymmetric $\mathrm{Al}$ rings, close to $T_{c}$ (see Fig. 4 in Ref. 22).

\section{CONCLUSIONS}

Concluding, dynamic properties of a rectangular superconducting loop with applied dc current through asymmetrically placed leads and in a perpendicular field are studied within the GL formalism. In the absence of applied magnetic field and with increasing injected current, the asymmetric loop experiences different local criticality. However, it selfregulates by allowing vortex entry until the vortex currents equilibrate the current flows in the two branches of the loop. The condition for the transition to the resistive state is found to be the equilibration of the momentum of the superconducting condensate in the two branches.

In the constant current regime, vortices enter the sample one by one with increasing field due to flux quantization; we show that this leads to finite jumps in the current density in both arms of the loop but also to finite jumps in the measured 
voltage between the normal-metal leads where current is injected. As we explain, this can be used as a new technique for monitoring the vortex state in mesoscopic superconductors by transport measurements. The asymmetric biasing also leads to a current polarity-dependent response of the sample to applied magnetic field. This stems from the favorable compensation of the vortex and the injected currents, and leads to an observable shift and a different behavior of the $V(H)$ characteristics for the two directions of applied current, both related to recent experimental efforts in the field. ${ }^{16,22}$

\section{ACKNOWLEDGMENTS}

This work was supported by the Flemish Science Foundation (FWO-Vl), the Belgian Science Policy (IAP), the ESF-NES program, and the ESF-AQDJJ network. G.R.B. acknowledges support from FWO-Vlaanderen. The authors thank S. Michotte for useful discussions. *francois.peeters@ua.ac.be

${ }^{1}$ B. S. Deaver, Jr. and W. M. Fairbank, Phys. Rev. Lett. 7, 43 (1961); R. Doll and M. Näbauer, ibid. 7, 51 (1961).

${ }^{2}$ A. H. Silver and J. E. Zimmerman, Phys. Rev. 157, 317 (1967).

${ }^{3}$ W. A. Little and R. D. Parks, Phys. Rev. Lett. 9, 9 (1962); R. D. Parks and W. A. Little, Phys. Rev. 133, A97 (1964).

${ }^{4}$ M. Baert, V. V. Metlushko, R. Jonckheere, V. V. Moshchalkov, and Y. Bruynseraede, Phys. Rev. Lett. 74, 3269 (1995); Q. Niu and F. Nori, Phys. Rev. B 39, 2134 (1989); G. R. Berdiyorov, M. V. Milošević, and F. M. Peeters, Phys. Rev. Lett. 96, 207001 (2006); Phys. Rev. B 74, 174512 (2006); G. R. Berdiyorov, B. J. Baelus, M. V. Milošević, and F. M. Peeters, ibid. 68, 174521 (2003).

${ }^{5}$ B. J. Baelus, F. M. Peeters, and V. A. Schweigert, Phys. Rev. B 61, 9734 (2000); S. V. Yampolskii, F. M. Peeters, B. J. Baelus, and H. J. Fink, ibid. 64, 052504 (2001); R. M. Arutunian and G. F. Zharkov, J. Low Temp. Phys. 52, 409 (1983); G. R. Berdiyorov, M. V. Milošević, B. J. Baelus, and F. M. Peeters, Phys. Rev. B 70, 024508 (2004).

${ }^{6}$ V. A. Schweigert, F. M. Peeters, and P. S. Deo, Phys. Rev. Lett. 81, 2783 (1998); B. J. Baelus and F. M. Peeters, Phys. Rev. B 65, 104515 (2002); J. Bonča and V. V. Kabanov, ibid. 65, 012509 (2001); A. S. Mel'nikov and V. M. Vinokur, Nature (London) 415, 60 (2002); L. F. Chibotaru, A. Ceulemans, V. Bruyndoncx, and V. V. Moshchalkov, ibid. 408, 833 (2000); R. Geurts, M. V. Milošević, and F. M. Peeters, Phys. Rev. Lett. 97, 137002 (2006).

${ }^{7}$ S. Pedersen, G. R. Kofod, J. C. Hollingbery, C. B. Sorensen, and P. E. Lindelof, Phys. Rev. B 64, 104522 (2001).

${ }^{8}$ J. R. Kirtley, C. C. Tsuei, V. G. Kogan, J. R. Clem, H. Raffy, and
Z. Z. Li, Phys. Rev. B 68, 214505 (2003).

${ }^{9}$ M. Morelle, D. S. Golubovic, and V. V. Moshchalkov, Phys. Rev. B 70, 144528 (2004).

${ }^{10}$ D. Yu. Vodolazov and F. M. Peeters, Phys. Rev. B 66, 054537 (2002); D. Yu. Vodolazov, F. M. Peeters, S. V. Dubonos, and A. K. Geim, ibid. 67, 054506 (2003).

${ }^{11}$ M. V. Milošević, A. Kanda, S. Hatsumi, F. M. Peeters, and Y. Ootuka, Phys. Rev. Lett. 103, 217003 (2009).

${ }^{12}$ A. Weber and L. Kramer, J. Low Temp. Phys. 84, 289 (1991).

${ }^{13}$ A. Th. A. M. de Waele, W. H. Kraan, R. de Bruyn Ouboter, and K. W. Taconis, Physica 37, 114 (1967).

${ }^{14} \mathrm{~S}$. Dubonos, V. Kuznetsov, and A. Nikulov, arXiv:physics/ 0105059 (unpublished).

${ }^{15}$ A. V. Nikulov, Phys. Rev. B 64, 012505 (2001).

${ }^{16}$ V. L. Gurtovoi, S. V. Dubonos, S. V. Karpii, A. V. Nikulov, and V. A. Tulin, Sov. Phys. JETP 105, 262 (2007).

${ }^{17}$ L. Kramer and R. J. Watts-Tobin, Phys. Rev. Lett. 40, 1041 (1978); R. J. Watts-Tobin, Y. Krähenbühl, and L. Kramer, J. Low Temp. Phys. 42, 459 (1981).

${ }^{18}$ G. R. Berdiyorov, M. V. Milošević, and F. M. Peeters, Phys. Rev. B 79, 184506 (2009).

${ }^{19}$ D. Yu. Vodolazov, D. S. Golubović, F. M. Peeters, and V. V. Moshchalkov, Phys. Rev. B 76, 134505 (2007).

${ }^{20}$ A. Kanda, B. J. Baelus, F. M. Peeters, K. Kadowaki, and Y. Ootuka, Phys. Rev. Lett. 93, 257002 (2004).

${ }^{21}$ D. S. Golubović, M. V. Milošević, F. M. Peeters, and V. V. Moshchalkov, Phys. Rev. B 71, 180502(R) (2005).

${ }^{22}$ A. A. Burlakov, V. L. Gurtovoi, S. V. Dubonos, A. V. Nikulov, and V. A. Tulin, JETP Lett. 86, 517 (2007). 